\title{
Family structure and affluence in adolescent eating behaviour: a cross-national study in forty-one countries
}

\author{
Apolinaras Zaborskis ${ }^{1 * *}$, Monika Grincaitè ${ }^{1}$ Aistè Kavaliauskiené ${ }^{2}$ and Riki Tesler ${ }^{3}$ \\ 'Faculty of Public Health, Lithuanian University of Health Sciences, A. Mickevičiaus str. 9, LT-44307 Kaunas, Lithuania: \\ ${ }^{2}$ Faculty of Odontology, Lithuanian University of Health Sciences, Kaunas, Lithuania: ${ }^{3}$ Department of Health Systems \\ Management, Faculty of Health Sciences, Ariel University, Ariel, Israel
}

Submitted 3 April 2020: Final revision received 19 August 2020: Accepted 10 September 2020: First published online 27 October 2020

\begin{abstract}
Objective: To investigate the family structure and affluence-related inequality in adolescent eating behaviour.

Design: Multivariate binary logistic regression and path analyses were employed to evaluate the impact of family structure and affluence on the consumption of fruits, vegetables, sweets and soft drinks among adolescents.

Setting: The cross-national Health Behaviour in School-aged Children study in 2013/2014 across forty-one countries.

Participants: Adolescents aged 11-15 years old ( $n 192$ 755).

Results: Adolescents from a non-intact family were less likely to eat daily fruits (OR $0.82 ; 95 \%$ CI 0.80, 0.84), vegetables (OR 0.91; $95 \%$ CI 0.89, 0.93) and sweets (OR 0.96; $95 \%$ CI 0.94, 0.99), but were more likely to consume soft drinks (OR 1.14; $95 \%$ CI 1.11, 1.17), compared with their counterparts from an intact family. Adolescents who had the lowest family affluence scores (FAS) were less likely to eat daily fruits (OR 0.51; $95 \%$ CI 0.49, 0.53), vegetables (OR 0.58; $95 \%$ CI $0.56,0.60)$ and sweets (OR 0.94; $95 \%$ CI 0.90, 0.97), but were more likely to consume soft drinks (OR 1.25; $95 \%$ CI 1.20, 1.30), compared with their counterparts who had the highest FAS. Across countries, a wide range of social inequality in daily consumption of foods was observed.

Conclusions: Among adolescents in Europe, Canada and Israel, there was a high level of family structure and family affluence inequalities in daily food consumption. Different aspects of family socio-economic circumstances should be considered at the national level designing effective interventions to promote healthy eating among adolescents.
\end{abstract}

Keywords
Adolescents
Eating habits
Social inequality
Health Behaviour in School-aged Children

Evidence gathered over the last few decades shows that relationships exist between socio-economic status (SES) and lifestyles of young people, including eating behaviour ${ }^{(1,2)}$. The influence of family affluence on adolescent eating habits has been the most extensively investigated $^{(3-6)}$. Studies show that adolescents living in high-affluence families consume healthier diets than counterparts living in low-affluence families. Family affluence may influence people's eating behaviour in several ways. In particular, differences in family affluence affect access to healthy food, and, therefore, adolescents from families of lower affluence report lower levels of fruit and vegetable consumption and often have nutrient and energy-poor diets ${ }^{(7,8)}$. Furthermore, living in low SES is often associated with low academic background of the parents that limits awareness of food nutritive value and healthy eating practice in family ${ }^{(9-11)}$. There are more possible explanations regarding SES level and the impact it may have on eating behaviour. One additional example includes the fact that individuals from high SES are more likely to uptake new behaviours ${ }^{(12)}$, for example, when healthy food is new, those from high SES will be the first to show such behaviour.

Family structure is another social factor with a tremendous impact on health in general and on children and adolescents in particular ${ }^{(13,14)}$. When evaluating the effect of divorce on health and well-being of children, the scientific research demonstrates that children with divorced parents consistently experience poorer physical, emotional and academic well-being than those living 
with their married biological parents ${ }^{(13,15-17)}$. Studies have also found that children living with a single parent are much more likely to live in poverty than children living with married parents ${ }^{(15,18)}$. Divorced parents often experience financial hardship due to the divorce, which can lead to the inability to obtain basic needs including adequate amounts of food, clothing and educational support. This type of family structure may also negatively affect children's nutrition; however, regardless of its wide range occurrence, there are few studies that examine the association between family structure and children's eating habits ${ }^{(19,20)}$. Formisano et al ${ }^{(19)}$ found that children who lived with their grandparents as opposed to both parents had higher BMI, while Mauskopf et al. ${ }^{(20)}$ found that preadolescents who had divorced parents consumed more sugar-sweetened drinks than those who lived with married parents. Mauskopf et al. ${ }^{(20)}$ also found that preadolescents with divorced parents consumed breakfast less frequently than those who had married parents.

Although studies have demonstrated that family affluence and structure are important factors in the nutrition of adolescents, to the best of our knowledge, the combined effect of both social factors on adolescent eating habits has not been studied at all. The analysis of this effect is limited by the interaction between social factors. As was mentioned above, it is likely that family structure without two married parents reduces family wealth; thus, the family structure affects adolescent eating habits both directly and indirectly due to family affluence. Indirectly, family affluence levels play a mediating role. The association between social factors and adolescent eating habits may vary depending on the social and cultural context, so it is important to examine the relationships among adolescents from different countries.

Social inequalities in health and health behaviours during adolescence is one of the foci of the cross-national Health Behaviour in School-aged Children (HBSC) study, which involves a wide network of researchers from more than forty countries and regions ${ }^{(1)}$. The previous editions of the study have highlighted the effect of socio-economic differences on the way young people grow and develop ${ }^{(1,2)}$. A significant positive association between family affluence and daily fruit consumption has been found for both genders and all three age groups (11, 13 and 15 year olds) in nearly all participating countries and regions, while the relationship between family affluence and soft drink consumption has not been consistent across countries.

The present study aimed to investigate the family structure and family affluence-related inequality in adolescent fruit, vegetable, sweets and chocolate, and soft drink consumption in forty-one HBSC (2013/2014) countries. A specific objective was to test the mediating effect of family affluence in the relationship between family structure variable and daily food consumption among adolescents.

\section{Methods}

\section{Subjects and study design}

The data were obtained from the HBSC study, a crossnational survey with support from the WHO (Europe), which was completed in 2013/2014 in forty-two countries, including forty European countries and regions (considered alone as countries, i.e., England, Scotland and Wales), Canada and Israel. More detailed background information about the study is provided on its website ${ }^{(21)}$ and in international reports ${ }^{(1,2)}$.

The population selected for sampling included 11-, 13- and 15-year-old adolescents. Sampling was conducted in accordance with the structure of national education systems within countries. In most countries, the primary sampling unit was the school class or the whole school where a sample frame of classes was not available. If a school with two or more classes was selected, then the one chosen for the sample was randomly selected.

The data were collected by means of self-report standardised questionnaires. The surveys were administrated in school classrooms. Students did not provide any personal details (such as name, classroom and teacher), making them completely anonymous and ensured the students' confidentiality. Researchers strictly followed the standardised international research protocol to ensure consistency in survey instruments, data collection and processing procedures $^{(22)}$. Response rates at the school, class and student level exceed $80 \%$ in the majority of countries ${ }^{(1)}$.

\section{Measures}

At the individual level, the outcome (dependent) variable was the frequency of selected food (fruit, vegetable, sweets and chocolate, or soft drink) consumption and the explanatory (independent) variables were family affluence, family structure, gender and age.

\section{Eating habits}

The frequency of four eating habits was assessed by questions: 'How many times a week do you consume fruits/vegetables/sweets and chocolate/soft drinks?' To make it more clear, in the survey provided to the students, there was a table with all of these options (fruits, vegetables, sweets and chocolate, soft drinks); students checked which ones they consume on a weekly basis and how many times per week: 'never' (1), 'less than once a week' (2), 'once a week' (3), '2-5 times a week' (4), '5-6 times a week' (5), 'once a day' (6) or 'more than once a day' (7). Daily consumption referred to eating these foods once or more times per day.

Family affluence was measured by the Family Affluence Scale ${ }^{(23,24)}$. The scale is a validated measure for material affluence of household based on the following six questions (assignment of points shown in parentheses): 'Does your family own a car, van, or truck?' ('no' (0), 'yes, one' (1), 'yes, two or more' (2)); 'Do you have your bedroom 
for yourself?' ('no' (0), 'yes' (1)); 'During the past 12 months, how many times did you travel away on holiday with your family?' ('not at all' (0), 'once' (1), 'twice' (2), 'more than twice' (3)); 'How many computers does your family own?' ('none' (0), 'one' (1), 'two' (2), 'more than two' (3)); 'How many bathrooms are in your home?' ('none' (0), 'one' (1), 'two' (2), 'three or more' (3)); 'Does your family have a dishwasher at home?' ('no' (0), 'yes' (1)). A family affluence score (FAS) was calculated by summing the points of the responses to these six questions. Higher FAS values indicated higher family affluence. In addition, this indicator was recoded into country-specific three groups. The first group included those in the lowest $20 \%$ (reference group), the second included those in the medium $60 \%$ and the third group included those in the highest $20 \%$ of the $\mathrm{FAS}^{(1)}$. The proportion of missing cases in the total sample for this scale was $8.3 \%^{(1)}$. The valid data on family affluence were obtained from forty-one countries (Armenia was excluded from the analysis due to deviance in the family affluence assessment).

\section{Family structure}

To identify family structure, respondents were given a checklist to mark the people living in their home. If the respondent ticked on the checklist that he or she lived with the biological mother and father, he or she was coded as living in 'intact family' ( 0 , reference value); in all other cases (meaning that not both mother and father were ticked), respondents were categorised as living in "non-intact family $^{\text {(1) }}$. On average, the proportion of missing cases in the total sample for family structure was $4.0 \%$.

\section{Gender and age}

Equal proportions of boys and girls, as well as 11-, 13- and 15-year-old adolescents were the target groups for the study.

\section{Statistical analysis}

All analyses were performed with SPSS (version 21.0; SPSS Inc.). They were conducted with data from all countries, as well as with data of each country. Statistics were estimated with $95 \%$ CI. Statistical tests with $P<0.05$ were considered statistically significant.

We first used an approach proposed by Kunst and Mackenbach to estimate the extent of SES-based inequality in health ${ }^{(25)}$. In this model, the ordinal FAS measure was transformed into a continuous $X$ variable scaled from 0 (the lowest FAS) to 1 (the highest FAS), incorporating appropriate population weights for each FAS category. In the present study, the binary logistic regression estimated the association between the continuous $X$ variable and proportion of daily food consumption. OR were derived from this model as a measure of health inequality, which denoted the ratio between the likelihood of daily food consumption at the lowest FAS level (at $X=0$ ) and at the highest FAS level (at $X=1)$. The logistic regression model included also a binary family structure variable to estimate effect (OR) of 'non-intact family' $v$. 'intact family' in daily food consumption. The models were adjusted for gender and age group variables. Then, we produced analyses of the total sample of all countries with weighting data by country sample size. The use of FAS in adolescent health inequality analysis was described in detail in previous research $^{(26,27)}$.

Next, path analysis was used to examine the hypothesised causal relationships of FAS and family structure with daily food consumption adjusting data for gender and age. These relationships were assumed to be unidirectional. Finally, structural equation modelling was conducted to assess the final model using a maximum likelihood estimation method, given its applicability to non-normal data ${ }^{(28-30)}$. The final model provided path coefficients $(\beta)$ showing the strength of the predictive relationship between the connected variables. The $\chi^{2}$ statistic was used to assess the magnitude of the discrepancy between the sample and fitted covariance matrices, where $P>0.05$ indicated that the model and data were consistent. Because this statistic is sensitive to sample size, model fit was primarily evaluated using the root mean square error of approximation and the comparative fit index. Root mean square error of approximation values lower than 0.05 indicated good fit and lower than 0.08 indicated reasonable fit. Comparative fit index values higher than 0.9 indicated adequate fit and higher than 0.8 indicated marginal $\mathrm{fit}^{(30)}$. Path analysis was performed using AMOS 21 (SPSS Inc.) ${ }^{(29)}$.

\section{Results}

\section{Socio-demographic characteristics and prevalence of eating behaviours}

A total of 192755 students from forty-one countries were eligible for the current study (Table 1). Of them, $48.3 \%$ were boys and $51.7 \%$ were girls. A negligible range between proportions of students' gender was common in all countries, except for Ireland and the Russian Federation, for which the difference between the percentages of boys and girls was more than 10 percentage points. In the whole sample, the age groups of 11,13 and 15 years achieved nearly equal proportions (31.3, 34.9 and $33.8 \%$, respectively). Across countries, there were nevertheless deviations, ranging from 24.0 to $40.0 \%$ in the youngest age group with similar patterns among 13 year olds and 15 year olds. In Slovakia, 11 year olds were not surveyed. The proportions of subjects in 'low', 'middle' and 'high' family affluence groups were relative for each country; across countries, they varied around 20, 60 and $20 \%$ groups, respectively. Family structure distribution showed wide variation across countries, ranging from $54.0 \%$ of adolescents living with both parents in Greenland to $93.2 \%$ in Albania ( $73.7 \%$ in the total sample). Table 1 displays the detailed information on socio-demographic characteristics of the participants in the current study, by countries. 
Table 1 Sample size $(n)$ and socio-demographic characteristics, by countries

\begin{tabular}{|c|c|c|c|c|c|c|c|c|c|c|c|}
\hline \multirow[b]{2}{*}{ Country } & \multirow[b]{2}{*}{$n$} & \multicolumn{2}{|c|}{ Gender (\%) } & \multicolumn{3}{|c|}{ Age (\%) } & \multicolumn{2}{|c|}{$\begin{array}{c}\text { Family } \\
\text { structure (\%) }\end{array}$} & \multicolumn{3}{|c|}{$\begin{array}{c}\text { Family } \\
\text { affluence (\%) }\end{array}$} \\
\hline & & Boys & Girls & $\begin{array}{c}11 \\
\text { year } \\
\text { old }\end{array}$ & $\begin{array}{c}13 \\
\text { year } \\
\text { old }\end{array}$ & $\begin{array}{c}15 \\
\text { year } \\
\text { old }\end{array}$ & $\begin{array}{l}\text { Intact } \\
\text { family }\end{array}$ & $\begin{array}{c}\text { Not } \\
\text { intact } \\
\text { family }\end{array}$ & Low & Middle & High \\
\hline Albania & 4849 & 48.5 & 51.5 & $32 \cdot 3$ & 33.2 & 34.4 & 93.2 & $6 \cdot 8$ & 23.9 & $55 \cdot 0$ & $21 \cdot 1$ \\
\hline Austria & 3114 & $46 \cdot 2$ & 53.8 & 31.7 & 30.9 & 37.4 & $75 \cdot 1$ & 24.9 & $20 \cdot 7$ & 57.7 & 21.6 \\
\hline $\begin{array}{l}\text { Belgium } \\
\text { (Flemish) }\end{array}$ & 3956 & 53.9 & $46 \cdot 1$ & $33 \cdot 3$ & $26 \cdot 1$ & $40 \cdot 6$ & $71 \cdot 0$ & $29 \cdot 0$ & 21.4 & $58 \cdot 0$ & $20 \cdot 6$ \\
\hline $\begin{array}{l}\text { Belgium } \\
\text { (French) }\end{array}$ & 5421 & $48 \cdot 9$ & $51 \cdot 1$ & $33 \cdot 6$ & $33 \cdot 3$ & $33 \cdot 2$ & $66 \cdot 5$ & $33 \cdot 5$ & $19 \cdot 1$ & $61 \cdot 6$ & $19 \cdot 3$ \\
\hline Bulgaria & 4282 & $50 \cdot 9$ & $49 \cdot 1$ & $32 \cdot 4$ & $32 \cdot 2$ & 35.4 & 74.7 & $25 \cdot 3$ & $20 \cdot 4$ & $62 \cdot 2$ & $17 \cdot 4$ \\
\hline Canada & 11234 & 48.0 & $52 \cdot 0$ & $24 \cdot 0$ & 37.6 & 38.3 & 65.9 & 34.1 & 20.9 & $58 \cdot 8$ & $20 \cdot 2$ \\
\hline Croatia & 4890 & 49.1 & $50 \cdot 9$ & $30 \cdot 6$ & 34.7 & 34.6 & 84.7 & $15 \cdot 3$ & $21 \cdot 0$ & $61 \cdot 0$ & $18 \cdot 0$ \\
\hline Czech Republic & 4824 & $47 \cdot 2$ & $52 \cdot 8$ & 30.7 & 33.9 & 35.4 & 68.0 & $32 \cdot 0$ & $19 \cdot 4$ & 60.5 & $20 \cdot 1$ \\
\hline Denmark & 3499 & $46 \cdot 3$ & 53.7 & 29.5 & $36 \cdot 0$ & 34.5 & $72 \cdot 2$ & 27.8 & 17.5 & 67.9 & $14 \cdot 7$ \\
\hline England & 4486 & $50 \cdot 1$ & 49.9 & $36 \cdot 2$ & $32 \cdot 2$ & 31.6 & 69.4 & $30 \cdot 6$ & $16 \cdot 4$ & 65.6 & $18 \cdot 0$ \\
\hline Estonia & 3965 & $50 \cdot 3$ & $49 \cdot 7$ & $32 \cdot 8$ & 35.4 & $31 \cdot 7$ & $65 \cdot 6$ & 34.4 & $20 \cdot 6$ & $57 \cdot 8$ & $21 \cdot 7$ \\
\hline Finland & 5693 & $48 \cdot 6$ & 51.4 & 33.7 & $32 \cdot 3$ & 34.0 & $70 \cdot 3$ & 29.7 & $16 \cdot 3$ & $65 \cdot 6$ & $18 \cdot 1$ \\
\hline France & 4996 & 49.1 & $50 \cdot 9$ & 28.7 & 39.1 & $32 \cdot 2$ & $70 \cdot 0$ & $30 \cdot 0$ & $16 \cdot 7$ & $61 \cdot 3$ & $22 \cdot 0$ \\
\hline Germany & 5585 & 50.5 & 49.5 & 27.9 & 35.4 & $36 \cdot 6$ & 74.3 & $25 \cdot 7$ & $18 \cdot 3$ & $61 \cdot 7$ & $20 \cdot 0$ \\
\hline Greece & 4045 & $49 \cdot 6$ & $50 \cdot 4$ & $32 \cdot 8$ & $35 \cdot 0$ & $32 \cdot 2$ & 83.8 & $16 \cdot 2$ & $19 \cdot 4$ & $60 \cdot 8$ & $19 \cdot 8$ \\
\hline Greenland & 755 & $47 \cdot 8$ & $52 \cdot 2$ & $31 \cdot 0$ & 33.1 & $35 \cdot 9$ & 54.0 & $46 \cdot 0$ & $17 \cdot 2$ & $62 \cdot 1$ & $20 \cdot 7$ \\
\hline Hungary & 3795 & 49.4 & $50 \cdot 6$ & $36 \cdot 2$ & 34.9 & 28.9 & $69 \cdot 3$ & 30.7 & 19.7 & $59 \cdot 6$ & $20 \cdot 7$ \\
\hline Iceland & 9561 & $49 \cdot 3$ & $50 \cdot 7$ & 31.9 & $36 \cdot 6$ & 31.5 & 69.7 & $30 \cdot 3$ & 24.0 & $56 \cdot 2$ & $19 \cdot 8$ \\
\hline Ireland & 3608 & 37.7 & $62 \cdot 3$ & $26 \cdot 2$ & $36 \cdot 5$ & $37 \cdot 3$ & $77 \cdot 2$ & $22 \cdot 8$ & 17.9 & $60 \cdot 2$ & 21.9 \\
\hline Israel & 5919 & $48 \cdot 1$ & 51.9 & $39 \cdot 3$ & $30 \cdot 2$ & $30 \cdot 4$ & 84.7 & $15 \cdot 3$ & $19 \cdot 2$ & $59 \cdot 8$ & $21 \cdot 0$ \\
\hline Italy & 3882 & $49 \cdot 6$ & $50 \cdot 4$ & 33.1 & $35 \cdot 3$ & 31.7 & $82 \cdot 2$ & $17 \cdot 8$ & $19 \cdot \overline{7}$ & $60 \cdot 8$ & $19 \cdot 6$ \\
\hline Latvia & 5413 & $47 \cdot 1$ & $52 \cdot 9$ & $33 \cdot 3$ & 35.4 & 31.3 & 64.0 & $36 \cdot 0$ & $22 \cdot 7$ & 54.6 & $22 \cdot 7$ \\
\hline Lithuania & 5507 & $50 \cdot 0$ & $50 \cdot 0$ & $35 \cdot 1$ & $35 \cdot 0$ & 29.9 & $70 \cdot 4$ & $29 \cdot 6$ & $20 \cdot 6$ & 59.5 & $19 \cdot 9$ \\
\hline Luxembourg & 2933 & $46 \cdot 1$ & 53.9 & $29 \cdot 3$ & 35.1 & $35 \cdot 6$ & $70 \cdot 6$ & 29.4 & $21 \cdot 1$ & $59 \cdot 6$ & $19 \cdot 3$ \\
\hline Malta & 1996 & $49 \cdot 0$ & $51 \cdot 0$ & $35 \cdot 3$ & $36 \cdot 2$ & $28 \cdot 6$ & $85 \cdot 1$ & 14.9 & $19 \cdot 1$ & $64 \cdot 8$ & $16 \cdot 1$ \\
\hline Netherlands & 3983 & 48.7 & $51 \cdot 3$ & 32.4 & 34.8 & $32 \cdot 8$ & $76 \cdot 1$ & 23.9 & 18.4 & $66 \cdot 0$ & $15 \cdot 6$ \\
\hline $\begin{array}{l}\text { North } \\
\text { Macedonia }\end{array}$ & 3936 & $49 \cdot 2$ & $50 \cdot 8$ & 33.4 & $31 \cdot 6$ & $35 \cdot 0$ & $87 \cdot 2$ & $12 \cdot 8$ & $21 \cdot 3$ & $59 \cdot 0$ & $19 \cdot 7$ \\
\hline Norway & 2658 & 47.4 & $52 \cdot 6$ & $40 \cdot 0$ & 31.2 & $28 \cdot 8$ & 74.7 & $25 \cdot 3$ & $19 \cdot 1$ & 64.7 & $16 \cdot 1$ \\
\hline Poland & 4282 & $49 \cdot 0$ & $51 \cdot 0$ & 33.3 & 33.8 & 32.9 & $77 \cdot 8$ & $22 \cdot 2$ & $17 \cdot 7$ & $65 \cdot 3$ & $17 \cdot 0$ \\
\hline Portugal & 4359 & $47 \cdot 3$ & $52 \cdot 7$ & 30.9 & $40 \cdot 0$ & 29.2 & 73.1 & $26 \cdot 9$ & $19 \cdot 8$ & $59 \cdot 3$ & 20.9 \\
\hline $\begin{array}{l}\text { Republic of } \\
\text { Moldova }\end{array}$ & 4529 & $50 \cdot 1$ & $49 \cdot 9$ & 33.4 & 33.1 & $33 \cdot 5$ & $76 \cdot 9$ & $23 \cdot 1$ & $20 \cdot 6$ & $57 \cdot 1$ & $22 \cdot 2$ \\
\hline Romania & 3454 & $45 \cdot 8$ & 54.2 & 29.7 & $32 \cdot 3$ & 38.0 & 75.5 & 24.5 & 18.9 & $61 \cdot 1$ & 19.9 \\
\hline $\begin{array}{l}\text { Russian } \\
\text { Federation }\end{array}$ & 4275 & $43 \cdot 0$ & $57 \cdot 0$ & $29 \cdot 7$ & $38 \cdot 7$ & $31 \cdot 6$ & $67 \cdot 7$ & $32 \cdot 3$ & $19 \cdot 8$ & $60 \cdot 7$ & $19 \cdot 5$ \\
\hline Scotland & 5565 & $49 \cdot 2$ & $50 \cdot 8$ & $32 \cdot 0$ & $35 \cdot 7$ & $32 \cdot 3$ & $65 \cdot 6$ & 34.4 & $17 \cdot 8$ & 64.4 & $17 \cdot 8$ \\
\hline Slovakia & 3461 & $48 \cdot 0$ & $52 \cdot 0$ & & $56 \cdot 3$ & $43 \cdot 7$ & $76 \cdot 7$ & $23 \cdot 3$ & $19 \cdot 4$ & 61.5 & $19 \cdot 1$ \\
\hline Slovenia & 4686 & 48.5 & 51.5 & $32 \cdot 3$ & $35 \cdot 1$ & $32 \cdot 6$ & 79.7 & $20 \cdot 3$ & $22 \cdot 6$ & 59.4 & 17.9 \\
\hline Spain & 6822 & 47.9 & $52 \cdot 1$ & $25 \cdot 0$ & 39.9 & $35 \cdot 2$ & $79 \cdot 6$ & 20.4 & 21.4 & 57.9 & $20 \cdot 7$ \\
\hline Sweden & 7028 & 48.9 & $51 \cdot 1$ & 33.0 & $30 \cdot 0$ & $37 \cdot 1$ & $69 \cdot 1$ & 30.9 & $17 \cdot 4$ & $58 \cdot 3$ & $24 \cdot 3$ \\
\hline Switzerland & 6322 & $49 \cdot 2$ & $50 \cdot 8$ & 29.5 & $36 \cdot 1$ & 34.3 & 76.9 & $23 \cdot 1$ & $19 \cdot 7$ & $63 \cdot 0$ & $17 \cdot 3$ \\
\hline Ukraine & 4307 & $46 \cdot 4$ & 53.6 & $32 \cdot 4$ & $30 \cdot 8$ & $36 \cdot 8$ & $72 \cdot 5$ & 27.5 & 21.6 & 57.9 & 20.5 \\
\hline Wales & 4880 & $50 \cdot 4$ & 49.6 & 35.0 & $36 \cdot 8$ & $28 \cdot 2$ & 61.5 & 38.5 & 19.9 & 65.0 & $15 \cdot 1$ \\
\hline HBSC average* & 192755 & $48 \cdot 3$ & $51 \cdot 7$ & $31 \cdot 3$ & 34.9 & 33.8 & 73.7 & $26 \cdot 3$ & 19.7 & $60 \cdot 8$ & 19.5 \\
\hline
\end{tabular}

HBSC, Health Behaviour in School-aged Children.

*Data weighted by country sample size.

Findings presented in Table 2 show frequencies of daily fruit, vegetable, sweets and chocolate, and soft drink consumption, by countries. Table 3 describes the proportions reporting daily consumption of these items by gender, age group, family structure and family affluence, which were estimated from the total sample.

Overall, frequency of eating fruit at least once a day was $37.0 \%$; it was lowest (13.4\%) among students in Greenland and highest (50.5\%) among students in Albania. The average frequency of daily vegetable consumption $(36.0 \%)$ was similar to that of fruits, with a range of $21.2 \%$ in Spain to $56.6 \%$ in Belgium (French). Girls reported to eating both fruits and vegetables more frequently than boys. Daily fruit and vegetable consumption decreased with age.

On average, one in four respondents (24.7\%) reported to eating sweets and chocolate daily. Across countries, this proportion had a wide range of $2 \cdot 3-43 \cdot 3 \%$ among students 
Table 2 Daily consumption of selected foods, by country

\begin{tabular}{|c|c|c|c|c|}
\hline \multirow[b]{2}{*}{ Country } & \multicolumn{4}{|c|}{ Proportion (\%) of adolescent who consumed at least once a day } \\
\hline & Fruit & Vegetables & Sweets and chocolate & Soft drinks \\
\hline Albania & 50.5 & 37.9 & $35 \cdot 7$ & $27 \cdot 7$ \\
\hline Austria & $45 \cdot 7$ & $29 \cdot 5$ & $29 \cdot 4$ & $15 \cdot 6$ \\
\hline Belgium (Flemish) & $28 \cdot 7$ & $53 \cdot 8$ & $22 \cdot 4$ & $29 \cdot 1$ \\
\hline Belgium (French) & $49 \cdot 1$ & $56 \cdot 6$ & 40.5 & $36 \cdot 0$ \\
\hline Bulgaria & $36 \cdot 8$ & 41.5 & $43 \cdot 3$ & $34 \cdot 0$ \\
\hline Canada & $46 \cdot 4$ & $41 \cdot 6$ & $14 \cdot 3$ & $9 \cdot 9$ \\
\hline Croatia & $35 \cdot 9$ & $28 \cdot 2$ & $30 \cdot 6$ & $22 \cdot 6$ \\
\hline Czech Republic & $37 \cdot 1$ & $27 \cdot 2$ & 20.9 & 14.9 \\
\hline Denmark & 43.4 & 43.9 & $6 \cdot 6$ & 5.5 \\
\hline England & $38 \cdot 3$ & $42 \cdot 8$ & $22 \cdot 7$ & $12 \cdot 9$ \\
\hline Estonia & $31 \cdot 8$ & $24 \cdot 0$ & $24 \cdot 3$ & $6 \cdot 5$ \\
\hline Finland & 23.9 & $27 \cdot 8$ & 2.5 & $2 \cdot 6$ \\
\hline France & 35.9 & $41 \cdot 3$ & 23.4 & $25 \cdot 7$ \\
\hline Germany & $37 \cdot 0$ & $24 \cdot 7$ & $27 \cdot 0$ & $19 \cdot 8$ \\
\hline Greece & $32 \cdot 9$ & $34 \cdot 2$ & $15 \cdot 3$ & 4.6 \\
\hline Greenland & 13.4 & $40 \cdot 5$ & $26 \cdot 6$ & $29 \cdot 3$ \\
\hline Hungary & $36 \cdot 0$ & $31 \cdot 0$ & 33.3 & $30 \cdot 0$ \\
\hline Iceland & $38 \cdot 6$ & $30 \cdot 2$ & $2 \cdot 3$ & 4.4 \\
\hline Ireland & 41.5 & $45 \cdot 3$ & $26 \cdot 1$ & 11.1 \\
\hline Israel & 44.4 & $48 \cdot 6$ & 33.2 & $30 \cdot 2$ \\
\hline Italy & $37 \cdot 8$ & $26 \cdot 8$ & $28 \cdot 3$ & $16 \cdot 0$ \\
\hline Latvia & $26 \cdot 2$ & $25 \cdot 8$ & $28 \cdot 1$ & 6.4 \\
\hline Lithuania & $32 \cdot 6$ & $30 \cdot 7$ & $21 \cdot 7$ & $11 \cdot 3$ \\
\hline Luxembourg & 38.0 & 33.6 & $24 \cdot 2$ & 28.3 \\
\hline Malta & 37.7 & 29.5 & $23 \cdot 0$ & $36 \cdot 4$ \\
\hline Netherlands & $35 \cdot 3$ & $47 \cdot 2$ & $30 \cdot 5$ & $25 \cdot 6$ \\
\hline North Macedonia & 39.8 & $40 \cdot 8$ & 35.4 & $30 \cdot 0$ \\
\hline Norway & 39.4 & $36 \cdot 4$ & $4 \cdot 8$ & $5 \cdot 3$ \\
\hline Poland & 33.8 & $29 \cdot 3$ & $27 \cdot 6$ & $23 \cdot 0$ \\
\hline Portugal & $40 \cdot 9$ & $27 \cdot 7$ & 14.8 & $16 \cdot 9$ \\
\hline Republic of Moldova & $35 \cdot 8$ & 39.9 & $30 \cdot 2$ & $10 \cdot 7$ \\
\hline Romania & 37.5 & $34 \cdot 1$ & 38.9 & $24 \cdot 8$ \\
\hline Russian Federation & $35 \cdot 9$ & $35 \cdot 6$ & $32 \cdot 3$ & $10 \cdot 4$ \\
\hline Scotland & 38.9 & 38.7 & 33.8 & 22.5 \\
\hline Slovakia & 31.5 & $25 \cdot 9$ & 35.4 & $25 \cdot 7$ \\
\hline Slovenia & $39 \cdot 3$ & $26 \cdot 9$ & $15 \cdot 7$ & $6 \cdot 4$ \\
\hline Spain & 34.7 & 21.2 & $12 \cdot 8$ & 20.2 \\
\hline Sweden & $27 \cdot 1$ & 40.7 & 3.5 & 4.9 \\
\hline Switzerland & $47 \cdot 2$ & $45 \cdot 3$ & 28.9 & $27 \cdot 0$ \\
\hline Ukraine & $47 \cdot 4$ & 53.7 & $39 \cdot 3$ & 13.9 \\
\hline Wales & $32 \cdot 2$ & $34 \cdot 3$ & $22 \cdot 9$ & $19 \cdot 8$ \\
\hline HBSC average* & 37.0 & $36 \cdot 0$ & 24.7 & 18.5 \\
\hline
\end{tabular}

HBSC, Health Behaviour in School-aged Children.

${ }^{*}$ Data weighted by country sample size.

in Iceland and Bulgaria, respectively. Daily consumption of soft drinks was reported in $18.5 \%$ of adolescents on average; the lowest level of its consumption was found among students in Finland $(2.6 \%)$, while students in Malta reported the highest levels (36.4\%). Girls generally reported greater daily consumption of sweets and chocolate but a lower daily consumption of soft drinks than boys. Daily consumption of both these items increased with age.

\section{Social inequalities in eating behaviour}

Table 3 also represents a comparison of eating habits between levels of family affluence and family structures. Analysis of the total sample showed that students living in low-affluent families or in non-intact families reported a noticeable lower frequency of daily consumption of fruits and vegetables, along with a greater frequency of a daily consumption of soft drinks than among their counterparts. In regard to daily sweets and chocolate consumption, the difference between the groups was less pronounced.

The results from a multivariate logistic regression analysis of family affluence-related inequality in and family structure-related inequality in adolescent eating behaviour are presented in Tables 4 and 5, respectively. After controlling for gender and age in the model of total sample, students who lived in the lowest family affluence group were significantly less likely to consume fruits daily (OR 0.51; $95 \%$ CI $0 \cdot 49,0.53)$, vegetables daily (OR 0.58; $95 \%$ CI $0.56,0.60)$ and sweets and chocolate daily (OR 0.94; $95 \%$ CI 0.90, 0.97), but were significantly more likely to consume soft drinks daily (OR 1.25; $95 \%$ CI 1.20, 1.30), compared with 
Table 3 Daily fruit, vegetable, sweet and soft drink consumption, by gender, age group, family structure and family affluence: Health Behaviour in School-aged Children average based on equal weighting of each country

\begin{tabular}{|c|c|c|c|c|}
\hline & \multicolumn{4}{|c|}{ Daily consumption (\%) } \\
\hline & Fruit & Vegetable & Sweet and chocolate & Soft drink \\
\hline \multicolumn{5}{|l|}{ Gender } \\
\hline Boys (n 93 101) & 33.4 & $32 \cdot 3$ & $23 \cdot 1$ & $20 \cdot 9$ \\
\hline Girls (n 99654$)$ & $40 \cdot 3$ & $39 \cdot 1$ & $26 \cdot 1$ & $16 \cdot 4$ \\
\hline$P$-value & $<0.001$ & $<0.001$ & $<0.001$ & $<0.001$ \\
\hline \multicolumn{5}{|l|}{ Age group } \\
\hline 11 year olds ( $n 60332$ ) & $42 \cdot 9$ & 38.7 & 22.5 & $15 \cdot 6$ \\
\hline 13 year olds ( $n 67271)$ & $36 \cdot 0$ & $34 \cdot 8$ & $25 \cdot 7$ & $19 \cdot 7$ \\
\hline 15 year olds ( $n 65152$ ) & $32 \cdot 0$ & $34 \cdot 0$ & $25 \cdot 7$ & $20 \cdot 4$ \\
\hline$P$-value & $<0.001$ & $<0.001$ & $<0.001$ & $<0.001$ \\
\hline \multicolumn{5}{|l|}{ Family affluence } \\
\hline Low $(n 38551)$ & $31 \cdot 7$ & 31.5 & $24 \cdot 6$ & $20 \cdot 4$ \\
\hline Middle ( $n 116424)$ & $36 \cdot 3$ & 35.4 & $24 \cdot 6$ & $18 \cdot 1$ \\
\hline High (n 37 780) & $44 \cdot 6$ & 41.9 & $25 \cdot 6$ & $17 \cdot 2$ \\
\hline$P$-value & $<0.001$ & $<0.001$ & 0.001 & $<0.001$ \\
\hline \multicolumn{5}{|l|}{ Family structure } \\
\hline Intact family ( $n 142060)$ & 38.5 & $36 \cdot 7$ & $24 \cdot 7$ & $17 \cdot 9$ \\
\hline Non-intact family (n 50695 ) & $32 \cdot 2$ & $33 \cdot 4$ & $24 \cdot 1$ & $20 \cdot 2$ \\
\hline$P$-value & $<0.001$ & $<0.001$ & 0.002 & $<0.001$ \\
\hline
\end{tabular}

Table 4 OR of daily consumption of selected foods comparing adolescents from the lowest and highest family affluence group: Health Behaviour in School-aged Children (HBSC) average and countries with the lowest and highest OR value

\begin{tabular}{|c|c|c|c|c|c|}
\hline \multicolumn{3}{|c|}{ Countries with the lowest OR value } & \multicolumn{3}{|c|}{ Countries with the highest OR value } \\
\hline Country & OR & $95 \% \mathrm{Cl}$ & Country & OR & $95 \% \mathrm{Cl}$ \\
\hline Daily fruit consumption & & & HBSC average & 0.51 & $0.49,0.53^{\star * *} \dagger \ddagger$ \\
\hline Greenland & 0.14 & $0.06,0.32^{\star \star \star}$ & Israel & 0.79 & $0.61,1.03$ \\
\hline Ukraine & 0.28 & $0.22,0.34^{\star \star *}$ & Slovenia & 0.70 & $0.57,0.87^{\star *}$ \\
\hline Canada & 0.34 & $0.30,0.39^{\star * *}$ & Germany & 0.70 & $0.57,0.85^{\star \star}$ \\
\hline Daily vegetable consumption & & & HBSC average & 0.58 & $0.56,0.60^{\star * \star} \ddagger$ \\
\hline England & 0.31 & $0.25,0.39^{\star \star \star}$ & Malta & 1.75 & $1 \cdot 24,2 \cdot 49^{\star \star \star}$ \\
\hline Wales & 0.32 & $0.26,0.40^{\star \star *}$ & Romania & 1.25 & $0.97,1.61$ \\
\hline Greenland & 0.33 & $0.19,0.57^{\star \star \star}$ & Albania & 0.92 & $0.75,1.13$ \\
\hline Daily sweets and chocolate consumption & & & HBSC average & 0.94 & $0.90,0.97^{\star} \ddagger$ \\
\hline Ukraine & 0.47 & $0.38,0.59^{\star \star \star}$ & Hungary & 1.91 & $1 \cdot 50,2 \cdot 44^{\star \star \star}$ \\
\hline Republic of Moldova & 0.57 & $0.45,0.71^{\star \star \star}$ & Greenland & 1.55 & $0.86,2.78$ \\
\hline Russian Federation & 0.59 & $0.47,0.74^{\star * *}$ & Slovakia & 1.38 & $1.07,1.77^{\star}$ \\
\hline Daily soft drink consumption & & & HBSC average & 1.25 & $1 \cdot 20,1 \cdot 30^{\star * *} \ddagger$ \\
\hline Republic of Moldova & 0.45 & $0.32,0.63^{\star \star \star}$ & Belgium (Flemish) & 3.06 & $2 \cdot 38,3 \cdot 94^{\star \star \star}$ \\
\hline Ukraine & 0.54 & $0.40,0.74^{\star \star \star}$ & Greenland & 2.88 & $1 \cdot 61,5 \cdot 18^{\star \star \star}$ \\
\hline Albania & 0.61 & $0.49,0.77^{\star \star *}$ & Ireland & $2 \cdot 82$ & $1 \cdot 91,4 \cdot 15^{\star \star \star}$ \\
\hline
\end{tabular}

†Data weighted by country sample size.

$\ddagger$ Bolded values are HBSC average.

${ }^{\star} P<0.05 ;{ }^{\star \star} P<0.01 ;{ }^{* \star \star} P<0.001$

their counterparts who lived in the highest family affluence group. Students who lived in a non-intact family were significantly less likely to eat fruits daily (OR $0 \cdot 82 ; 95 \% \mathrm{CI} 0 \cdot 80$, $0.84)$, vegetables daily (OR 0.91; $95 \% \mathrm{CI} 0.89,0.93)$ and sweets and chocolate daily (OR 0.96; $95 \%$ CI 0.94, 0.99), but were significantly more likely to consume soft drinks daily (OR 1.14; $95 \%$ CI 1.11, 1.17), compared with those who lived in families with both parents.

Across countries, a wide range of social inequality in daily eating of selected foods was observed (Tables 4 and 5 present results of the current analysis only for three countries with the lowest and highest inequalities selecting for each food type). Low FAS was uniformly associated with lower levels of fruit consumption among students in all countries. The OR measure of inequality, which was used to estimate the impact of family affluence on daily fruit eating, ranged from 0.14 (95\% CI 0.06, 0.32) in Greenland to $0.79(95 \%$ CI $0.61,1.03)$ in Israel that was a distinguished country with not sufficient $P$-value of this association. Daily fruit consumption dropped significantly between intact and non-intact families in nineteen countries, and this tendency was seen in the majority of countries. A similar pattern was observed regarding vegetable consumption. Malta, however, stood out here, as the dependence of daily vegetable eating on family affluence was inverse than in most other countries. 
Table 5 OR of daily consumption of selected foods comparing adolescents from non-intact and intact family: Health Behaviour in School-aged Children (HBSC) average and countries with the lowest and highest OR value

\begin{tabular}{|c|c|c|c|c|c|}
\hline \multicolumn{3}{|c|}{ Countries with the lowest OR value } & \multicolumn{3}{|c|}{ Countries with the highest OR value } \\
\hline Country & OR & $95 \% \mathrm{Cl}$ & Country & OR & $95 \% \mathrm{Cl}$ \\
\hline Daily fruit consumption & & & HBSC average & 0.82 & $0.80,0.84^{\star \star \star} † \ddagger$ \\
\hline Scotland & 0.72 & $0.64,0.82^{\star \star \star}$ & Greece & $1 \cdot 10$ & $0.91,1.32$ \\
\hline Wales & 0.74 & $0.65,0.85^{\star \star \star}$ & Greenland & 1.02 & $0.65,1.58$ \\
\hline Spain & 0.75 & $0.66,0.85^{\star \star *}$ & Albania & 1.01 & $0.80,1.27$ \\
\hline Daily vegetable consumption & & & HBSC average & 0.91 & $0.89,0.93^{\star \star \star} \ddagger$ \\
\hline Germany & 0.71 & $0.61,0.82^{\star \star \star}$ & Malta & 1.27 & $0.97,1.66$ \\
\hline Scotland & 0.72 & $0.64,0.82^{\star \star \star}$ & Belgium (Flemish) & 1.14 & $0.99,1.32$ \\
\hline Wales & 0.76 & $0.66,0.89^{\star \star \star}$ & Slovenia & 1.09 & $0.92,1.28$ \\
\hline Daily sweets and chocolate consumption & & & HBSC average & 0.96 & $0.94,0.99^{\star} \ddagger$ \\
\hline Malta & 0.79 & $0.58,1.08$ & Iceland & 1.49 & $1.13,1.98^{\star \star \star}$ \\
\hline Netherlands & 0.80 & $0.68,0.95^{\star *}$ & Denmark & 1.39 & $1.04,1.86^{\star}$ \\
\hline Switzerland & 0.82 & $0.72,0.94^{*}$ & Greece & 1.36 & $1.09,1 \cdot 70^{\star \star}$ \\
\hline Daily soft drink consumption & & & HBSC average & 1.14 & $1 \cdot 11,1 \cdot 17^{\star * \star} \ddagger$ \\
\hline Switzerland & 0.97 & $0 \cdot 85,1 \cdot 11$ & Finland & 1.94 & $1 \cdot 38,2 \cdot 73^{\star \star \star}$ \\
\hline Hungary & 1.00 & $0 \cdot 86,1 \cdot 16$ & Ireland & 1.79 & $1 \cdot 42,2 \cdot 25^{\star \star \star}$ \\
\hline North Macedonia & 1.03 & $0.83,1.27$ & Norway & 1.70 & $1.19,2 \cdot 45^{\star \star \star}$ \\
\hline
\end{tabular}

†Data weighted by country sample size.

$\ddagger$ Bolded values are HBSC average.

${ }^{*} P<0.05 ;{ }^{* *} P<0.01 ;{ }^{* \star *} P<0.001$.

Daily eating of sweets and chocolate differed according to FAS and family structure in a few countries, but no consistent pattern emerged for these social factors. There were several countries (e.g., Ukraine and Netherlands) where students from low-affluence families and non-intact families reported less eating of sweets and chocolate daily, and there was a consistent group of countries (e.g., Hungary and Iceland) where the students from the same kind of families reported to more eating of sweets and chocolate daily, in comparison to their counterparts elsewhere.

Soft drink consumption was significantly associated with FAS in approximately half of the countries surveyed; however, the value of this association varied noticeable across countries. There was a group of countries where the daily consumption of soft drinks was associated with high FAS; this pattern was strongest in Eastern Europe and the Baltic states. The reverse pattern was found in western and northern Europe; here the daily consumption of soft drinks was associated with low-affluence families. The frequency of daily soft drink consumption was higher among students from non-intact families compared with students from intact families in the majority of countries, and in around two-thirds of these countries a significant difference was detected.

An overview of the presented figures above indicates the possibility of correlation between estimated inequalities. Results from the Spearman correlation analysis of these estimations are presented in Table 6 . With respect to inequalities due to family affluence, significant positive correlations were observed between inequalities in daily fruit and vegetable consumption, as well as between inequalities in daily sweets and chocolate consumption and daily soft drink consumption. In contrast, family affluence defined inequalities in daily vegetable and soft drink consumption were negatively correlated; this indicated that countries with
Table 6 Spearman correlations between inequalities in fruit, vegetable, sweets and chocolate and soft drink consumption among adolescents in forty-one Health Behaviour in School-aged Children countries

\begin{tabular}{|c|c|c|c|c|}
\hline & Fruit & Vegetable & $\begin{array}{l}\text { Sweets and } \\
\text { chocolate }\end{array}$ & $\begin{array}{l}\text { Soft } \\
\text { drink }\end{array}$ \\
\hline Fruit & & $0.612^{\star \star \star}$ & -0.181 & $\begin{array}{l}-0.234 \\
-0.178\end{array}$ \\
\hline $\begin{array}{r}\text { Sweets and } \\
\text { chocolate }\end{array}$ & 0.034 & $-0 \cdot 180$ & -0.401 & $0.500^{\star * *}$ \\
\hline Soft drinks & 0.022 & $-0.528^{\star \star \star}$ & $0.582^{* \star \star}$ & \\
\hline
\end{tabular}

An upper triangle represents inequalities due to family structure, and lower triangle represents inequalities due to family affluence.

${ }^{\star} P<0.05 ;{ }^{* \star} P<0.01 ;{ }^{\star \star *} P<0.001$.

a high inequality in vegetable consumption had a low inequality in soft drinks consumption. With respect to inequalities due to family structure, significant positive correlations were found between the same correlates as for the inequalities caused by family affluence, but the negative correlation was observed between inequalities in daily vegetable consumption and daily sweet and chocolate consumption. Any significant correlations were found between inequalities defined by family affluence and inequalities defined by family structure (results not shown).

\section{Path analysis}

The path models were studied for daily consumption of each food type. Figure 1 represents an example of such models with assessments of the standardised regression weights that were calculated for daily fruit consumption from the total sample of the forty-one countries. Table 7 shows corresponding assessments and the goodness-of-fit statistics of models for remaining food types. 


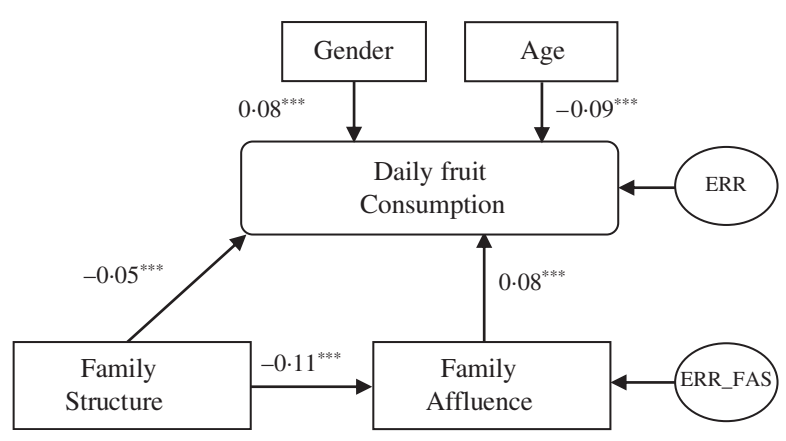

Fig. 1 Path model of daily fruit consumption. Arrows indicate a direction of the association; the numbers are standardised regression weights $(\beta)$. Estimations were done from the total sample of forty-one countries. ERR and ERR_FAS are other determinants that affect corresponding variables. ${ }^{\star \star *} P<0.001$

Using the total sample of forty-one countries, the results of the path analysis were consistent with the data presented in Table 3. Standardised regression weights $(\beta)$ indicated that girls were more likely than boys to report consuming fruits, vegetables, and sweets and chocolate daily but less likely to report consuming soft drinks. By age, fruit and vegetable consumption decreased but sweets and chocolate, as well as soft drink consumption increased. The non-intact family structure had a direct negative impact on daily fruit, vegetable, and sweets and chocolate consumption, but its effect was positive on daily soft drink consumption. FAS had a positive influence on daily fruit, vegetable, and sweets and chocolate consumption (e.g., if the FAS increased, daily consumption of these foods increased), but the corresponding relationship was inverse for soft drink consumption. In addition to these common findings, the path analysis revealed that a non-intact family, compared with an intact family, reduced FAS on average by $11 \%(\beta=-0 \cdot 11)$. In some countries (e.g., Scotland) this effect reached up to $26 \%$. Therefore, family structure was hypothesised to exert an indirect effect on daily food consumption through family affluence.

We were interested in the extent to which these models fit country data and the impact of each social factor on the inequality in daily food consumption for an individual country. For this purpose, from Tables 4 and 5 , the countries that exhibited the highest and lowest social inequalities in daily food consumption were selected. Results from path analysis for these countries are shown in Table 6. In Greenland, the high inequality of daily fruit consumption was only due to differences in family affluence, whereas changes in family structure did not significantly influence the inequality; such a model was simple and well fit to national data. In Scotland, high inequality in daily fruit consumption due to family structure was observed; it can be seen that this inequality was due not only to the direct but also to the strong indirect effect of changes in family structure on the child's nutrition. A similar pattern was observed in Germany's data, explaining the high inequality in daily vegetable consumption related to changes in family structure. Considering soft drink consumption among students in the Republic of Moldova and Belgium (Flemish), non-intact families had the same consequences in both countries; therefore, the inequality in the consumption of soft drinks was related mainly to family affluence. The influence on daily soft drink consumption had opposite signs in the Republic of Moldova and Belgium (Flemish). The consumption of soft drinks among Swiss students was not related to family structure or family affluence. In Finland, inequality in soft drink consumption was strongly related to a non-intact family structure, although the latter factor reduced FAS.

The goodness-of-fit statistics for the models are included in Table 7. Due to the large sample size, the $\chi^{2}$ statistic that assessed the magnitude of the discrepancy between the sample and fitted covariance matrices for several models indicated that the model and data were consistent $(P>0.05)$. However, for all path models tested in the current study, the main statistic RMSEA showed an acceptable model fit (RMSEA $<0.08$ ), and it indicated a very good fit for the majority of the models (RMSEA $<0.05$ ). For most models, comparative fit index statistics also showed an acceptable fit (comparative fit index $>0.9$ ) or was close to the threshold.

\section{Discussion}

The current study examined family structure and family affluence-related inequalities in the consumption of fruits, vegetables, sweets and chocolate, and soft drinks among adolescents in forty-one countries. Inadequate or insufficient intake of fruits and vegetables was common among the study population. Many adolescents also ate sweets and drank soft drinks regularly. On average, only one-third of the adolescents reported consuming fruits and vegetables at least once a day, one in four respondents reported eating sweets and chocolate daily and one in five respondents reported drinking soft drinks daily.

Our results showed that adolescents from non-intact families were less likely to report daily fruit and vegetable consumption in almost all of the study countries, and in nearly half of the countries this effect was significant when compared with adolescents living with both parents. Such a result is logical, since coming from a non-intact family negatively affects family affluence, which, as we have seen, is directly related to the consumption of fruits and vegetables $^{(31-35)}$. The literature on the impact of the non-intact family on eating fruits and vegetables in adolescents is very scarce. The study by Pearson et al. ${ }^{(36)}$ among adolescents in the United Kingdom (England) revealed no significant association between family structure and any dietary behaviours. We also found several countries where no such association was found, but in the aforementioned England, non-intact family had a significant negative impact on adolescent fruit and vegetable consumption. Our study also 
Table 7 Standardised regression weights and model goodness-of-fit statistics in path models of daily fruit, vegetable, sweets and chocolate, and soft drink consumption in selected and all Health Behaviour in School-aged Children (HBSC) countries

\begin{tabular}{|c|c|c|c|c|c|c|c|c|}
\hline & \multicolumn{5}{|c|}{ Standardised regression weights $(\beta)$} & \multicolumn{3}{|c|}{ Model goodness-of-fit statistics } \\
\hline & $\mathrm{FS} \rightarrow \mathrm{FAS}$ & Gender $\rightarrow$ DFC & Age $\rightarrow$ DFC & $\mathrm{FS} \rightarrow \mathrm{DFC}$ & $\mathrm{FAS} \rightarrow \mathrm{DFC}$ & $\chi^{2} / \mathrm{df}$ & $\mathrm{CFI}$ & RMSEA \\
\hline \multicolumn{9}{|c|}{ Models of daily fruit consumption } \\
\hline Greenland & -0.05 & 0.06 & -0.06 & 0 & $0 \cdot 17^{\star \star \star}$ & 1.173 & 0.957 & 0.013 \\
\hline Israel & $-0 \cdot 14^{\star \star \star}$ & $0 \cdot 11^{\star \star *}$ & $-0.05^{\star \star}$ & -0.02 & 0.03 & $11 \cdot 37^{\star \star \star}$ & 0.746 & 0.041 \\
\hline Scotland & $-0.26^{\star \star *}$ & $0.07^{\star \star \star}$ & $-0 \cdot 13^{\star \star *}$ & $-0.06^{\star * \star}$ & $0 \cdot 13^{\star \star \star}$ & $3.519^{\star \star}$ & 0.981 & 0.021 \\
\hline Greece & $-0.07^{\star \star *}$ & $0.05^{\star \star}$ & $-0 \cdot 13^{\star \star *}$ & 0.01 & $0.06^{\star \star \star}$ & $4 \cdot 170^{\star \star \star}$ & 0.863 & 0.028 \\
\hline HBSC average & $-0.11^{\star \star *}$ & $0.08^{\star * *}$ & $-0.09^{\star \star \star}$ & $-0.05^{\star \star \star}$ & $0.08^{\star * *}$ & $144 \cdot 6^{\star \star \star}$ & 0.910 & 0.026 \\
\hline \multicolumn{9}{|c|}{ Models of daily vegetable consumption } \\
\hline England & $-0 \cdot 22^{\star \star \star}$ & $0.06^{\star \star \star}$ & $-0.03^{*}$ & $-0.03^{*}$ & $0 \cdot 16^{\star \star \star}$ & $8.033^{\star \star \star}$ & 0.912 & 0.036 \\
\hline Malta & $-0 \cdot 16^{\star * \star}$ & $-0 \cdot 13^{\star \star \star}$ & $0.08^{\star \star *}$ & 0.03 & $-0.07^{\star *}$ & $3 \cdot 173^{\star \star}$ & 0.904 & 0.031 \\
\hline Germany & $-0 \cdot 16^{\star \star *}$ & $0.13^{\star \star \star}$ & $-0.03^{\star *}$ & $-0.06^{\star \star *}$ & 0.01 & $14 \cdot 70^{\star \star *}$ & 0.799 & 0.048 \\
\hline Malta & $-0 \cdot 16^{\star \star \star}$ & $-0 \cdot 13^{\star \star \star}$ & $0.08^{\star \star *}$ & 0.03 & $-0.07^{* *}$ & $3 \cdot 173^{\star \star}$ & 0.904 & 0.031 \\
\hline HBSC average & $-0 \cdot 11^{\star \star \star}$ & $0.08^{\star \star \star}$ & $-0.04^{\star \star \star}$ & $-0.03^{\star \star \star}$ & $0.07^{\star \star \star}$ & $144 \cdot 1^{\star \star \star}$ & 0.879 & 0.026 \\
\hline \multicolumn{9}{|c|}{ Models of daily sweets and chocolate consumption } \\
\hline Ukraine & $-0 \cdot 13^{\star \star \star}$ & $0.08^{* \star *}$ & 0.02 & 0 & $0 \cdot 10^{\star \star *}$ & $14 \cdot 27^{\star \star *}$ & 0.673 & 0.054 \\
\hline Hungary & $-0.09^{\star \star \star}$ & $0.04^{*}$ & 0.01 & -0.01 & $-0.09^{\star * *}$ & $11.48^{\star * *}$ & 0.527 & 0.052 \\
\hline Malta & $-0 \cdot 16^{\star \star \star}$ & $0.06^{\star \star}$ & $-0.04^{*}$ & -0.03 & 0.03 & $3 \cdot 158^{\star \star}$ & 0.839 & 0.031 \\
\hline Iceland & $-0 \cdot 17^{\star \star \star}$ & -0.02 & 0.01 & 0.02 & -0.01 & $6 \cdot 733^{\star \star \star}$ & 0.901 & 0.023 \\
\hline HBSC average & $-0 \cdot 11^{\star \star *}$ & $0.03^{\star \star \star}$ & $0.03^{\star \star \star}$ & $-0.02^{\star *}$ & $-0.06^{\star \star *}$ & $144 \cdot 3^{\star \star \star}$ & 0.835 & 0.026 \\
\hline \multicolumn{9}{|c|}{ Models of daily soft drink consumption } \\
\hline Republic of Moldova & $-0 \cdot 12^{\star \star \star}$ & $-0.03^{*}$ & 0 & $0.03^{*}$ & $0.07^{* \star *}$ & $10 \cdot 16^{\star * *}$ & 0.660 & 0.044 \\
\hline Belgium (Flemish) & $-0 \cdot 15^{\star \star \star}$ & $-0.09^{\star \star \star}$ & $0.09^{\star \star \star}$ & $0.04^{\star *}$ & $-0 \cdot 14^{\star \star \star}$ & $12 \cdot 59^{\star \star \star}$ & 0.807 & 0.051 \\
\hline Switzerland & $-0 \cdot 14^{\star \star \star}$ & $-0.09^{\star \star \star}$ & $0.06^{\star \star \star}$ & 0 & -0.02 & $3.519^{\star \star}$ & 0.981 & 0.021 \\
\hline Finland & $-0 \cdot 15^{\star \star \star}$ & $-0.09^{\star \star *}$ & $0.03^{\star}$ & $0.05^{\star \star *}$ & 0 & 1.056 & 0.999 & 0.003 \\
\hline HBSC average & $-0.11^{\star \star \star}$ & $-0.06^{\star \star \star}$ & $0.04^{\star \star *}$ & $0.02^{\star *}$ & $-0.03^{\star * *}$ & $144 \cdot 1^{* * *}$ & 0.844 & 0.026 \\
\hline
\end{tabular}

FS, family structure; FAS, family affluence score; DFC, daily food consumption; CFI, comparative fit index; RMSEA, root mean square error of approximation.

${ }^{*} P<0.05 ;{ }^{* *} P<0.01 ;{ }^{* \star *} P<0.001$.

showed that adolescents from non-intact families were more likely to drink soft drinks daily than their peers living with both parents; this pattern was significant in more than half of the countries. These findings were in line with the findings of Mauskopf's et al. study ${ }^{(20)}$, which showed that preadolescents with divorced parents consumed more sugar-sweetened beverages than preadolescents with married parents.

Family affluence also had a significant influence on adolescent eating habits, and it was, therefore, appropriate to evaluate their related inequalities regarding adolescent nutrition. This approach was based on the assumption that the relationship of adolescent nutrition and socio-demographic factors depended on the specific socio-cultural context ${ }^{(37-40)}$. It has been well documented in the literature ${ }^{(3-6)}$ that adolescents living in families with low SES consume less healthy diets that their counterparts living in families with high SES. Results from our study were in line with such conclusions in regard to fruit and vegetable consumption, showing daily intake of these foods less frequently among students from lowaffluent families in almost all countries. Although there was a great variety to the degrees of inequality in fruit and vegetables consumption, there were no clear geographic patterns in such inequalities. It is also difficult to explain why in Malta alone students from low-affluence families were more likely to report regular eating of vegetables compared with their counterparts from highly affluent families. It is also interesting to note that family affluence-related inequalities in daily fruit and vegetable intake correlated positively, that is, in countries with high inequality in fruit eating, there was also significant inequality in vegetable eating. The family affluence-related inequality in daily sweets and chocolate, as well as soft drink intake, was not consistent across countries, but at the country level, a significant correlation between their values was identified. There were some countries where adolescents from low-affluence families consumed these foods less frequently, while there were also some countries with an inverse relationship. There were no clear geographic patterns for inequality in sweets and chocolate consumption, but inequality in soft drinks consumption differed noticeably between countries in Eastern Europe and the Baltic states (high intake in high-affluence families) and countries in Western and Northern Europe (high intake in low-affluence families). The pattern identified may be partially associated with availability and pricing contrasts of foodstuffs $^{(31,41,42)}$. Soft drinks were among the first western goods to break into Eastern Europe and the Baltic states in the post-Soviet period, and yet their impact on health issues has not been appropriately discussed in health education in schools in these countries. This assumption is consistent with the strong negative correlation found at the country level between soft drink and vegetable consumption inequalities due to family affluence. 
Gender and age differences were similar to other studies $^{(1,2,43,44)}$, in the sense that healthier consumption habits occurred among the girls as they more often ate fruits and vegetables and consumed less soft drinks. Adolescents' eating habits worsened with age; the frequency of eating fruits and vegetables daily decreased with age, whereas the frequency of eating sweets and drinking soft drinks daily increased with age.

Finally, the path model was helpful in providing a more comprehensive understanding of the relationships between social determinants and allowed an assessment of the impact of each determinant on adolescent eating behaviour. The analysis showed that in most of the countries, dietary inequality was more pronounced for family affluence than for family structure. Moreover, family affluence was hypothesised to mediate the relationship between family structure and adolescent eating habits. This hypothesis was confirmed in all national samples and in the total sample of all countries. One possible explanation for mediating pathway is the negative influence of non-intact family on the material and psychological wealth of the family. Such an influence was confirmed in different studies ${ }^{(13,15-18)}$. There is not much literature on adolescent dietary studies using path analysis. For example, Pettigrew et al. ${ }^{(45)}$ used this method to elucidate factors that increase the propensity of children to consume unhealthy food. Another study ${ }^{(46)}$ investigated the complex effect of family income and parental education on a child's nutrition; its results showed that fruit and vegetable consumption was only related to family income.

Overall, the results of the present research are a step forward to filling the gap of mapping health inequalities in the context of social determinants in youth. Particularly, they provided a better understanding of the mechanism that links family structure and family affluence and how it influences adolescent eating behaviour. Different initiatives for reduction of family structure and family affluence-related inequalities should therefore be considered in order to make effective interventions to promote healthy eating in the young population.

\section{Methodological considerations}

The current study had several strengths. First, it utilises data from the HBSC survey, which collects longitudinal data worldwide on adolescent behaviour. Another strength is the fact that data from forty-one countries are analysed in the current study, which allows for a broader understanding of eating behaviour among adolescents worldwide. Although the sample size and methodology of the current analysis strengthen the findings, there are several limitations to the study. First, because of the cross-sectional design of the HBSC study, our study is limited in terms of its potential to establish causal inferences about the relationships observed. Longitudinal studies should be conducted in order to expand the understanding of the relationship between social determinants and eating behaviour outcomes. Second, food choice may be influenced by a number of additional social and economic factors, such as parental occupation, education level and place of residence (urban/rural) that were not available in our data. It is worthwhile to explore these factors in future studies on social family environment-related inequality in adolescent eating behaviour. Third, food consumption data were collected using a self-reported FFQ. In this measure, adolescents may have overreported consumption of healthy foods and underreported consumption of unhealthy products due to social pressure ${ }^{(47)}$. The use of self-report data on socio-economic characteristics may also have introduced response bias that we were not able to account for. The analysis was still controled by gender and age, as only two of these counfounders were available in all country data. Finally, in path analysis we were interested in the relative strengths of coefficients. However, path analysis will correctly estimate the relative strengths of competing factors only for the model under consideration. While such analysis offers structured models that may underlie hypotheses, it cannot by itself confirm or disprove the existence of causal relationship ${ }^{(28)}$.

\section{Conclusions}

Among adolescents in European countries, Canada and Israel, there was a high level of family structure and family affluence inequalities in daily food consumption. In most countries, adolescents living in non-intact and low-affluent families were less likely to consume fruits and vegetables daily. With regard to daily consumption of sweets and chocolate, as well as soft drinks, such a pattern was not consistent.

Overall, the results of the present study provided a better understanding of the mechanism that links family structure and family affluence and how it influences eating behaviour among adolescents across different countries. Therefore, country-specific initiatives for reduction of family structure and family affluence-related inequalities should be considered in national policy in order to make effective interventions to promote healthy eating in the young population. Future research could be used to assess the impacts of other family determinants on adolescent eating behaviour in cross-national perspective, and ideally with longitudinal studies.

\section{Acknowledgements}

Acknowledgements: The HBSC study is an international study carried out in collaboration with WHO (Europe). Professor Candace Currie from the University of St. Andrews, United Kingdom served as the international 
coordinator of the 2013-2014 survey, and Professor Oddrun Samdal from Bergen University, Norway served as the international databank manager. The principal investigators in each of the forty-one countries were responsible for the HBSC survey undertaken in their country. Financial support: Data collection was funded by each of the participating countries and regions separately. The analysis of data and preparation of the manuscript received no specific grant from any funding agency, commercial or not-forprofit sectors. Conflict of interest: The authors declare that they have no competing interests. Authorship: A.Z. made substantive intellectual contribution to the conception and design of the manuscript and carried out the statistical analysis. M.G. was involved in national data collection and carried out the drafting of the manuscript. A.K. conducted a literature analysis, which is included in the manuscript. R.T. substantially improved the content and provided the final edition of the manuscript. All authors read and approved the final manuscript. Ethics of human subject participation: The current study was conducted in accordance with the Declaration of Helsinki. All procedures, involving research study participants, were approved by each national ethics committees according to the national guidance and regulations at the time of data collection. Additionally, written informed consent for participation in the study was sought from parents of the adolescents.

\section{References}

1. Inchley J, Currie D, Young T et al. (editors) (2016) Growing up Unequal: Gender and Socioeconomic Differences in Young People's Health and Well-being. Health Behaviour in School-aged Children (HBSC) Study: International Report from the 2013/2014 Survey. Copenhagen: World Health Organization Regional Office for Europe (Health Policy for Children and Adolescents, No. 7).

2. Currie C, Zanotti C, Morgan A et al. (editors) (2012) Social Determinants of Health and Well-being among Young People. Health Behaviour in School-aged Children (HBSC) Study: International Report from the 2009/2010 Survey. Copenhagen: World Health Organization Regional Office for Europe (Health Policy for Children and Adolescents, No. 6).

3. Voráčová J, Sigmund E, Sigmundová D et al. (2016) Family affluence and the eating habits of 11- to 15-year-old Czech adolescents: HBSC 2002 and 2014. Int J Environ Res Public Health 13, 1034.

4. Fismen AS, Samdal O \& Torsheim T (2012) Family affluence and cultural capital as indicators of social inequalities in adolescent's eating behaviours: a population-based survey. BMC Public Health 12, 1036.

5. Fismen AS, Smith OR, Torsheim T et al. (2016) Trends in food habits and their relation to socioeconomic status among Nordic adolescents 2001/2002-2009/2010. PLoS One 11, 0148541.

6. Zaborskis A, Lagunaite R, Busha R et al. (2012) Trend in eating habits among Lithuanian school-aged children in context of social inequality: three cross-sectional surveys 2002, 2006 and 2010. BMC Public Health 12, 52.
7. Gaspar T, Matos M, Ribeiro J et al. (2009) Health-related quality of life in children and adolescents and associated factors. J Cogn Behav Psychol 9, 33-48.

8. Wang Y (2001) Cross-national comparison of childhood obesity: the epidemic and the relationship between obesity and socioeconomic status. Int J Epidemiol 30, 1129-1136.

9. He L, Zhai Y, Engelgau M et al. (2014) Association of children's eating behaviors with parental education, and teachers' health awareness, attitudes and behaviors: a national schoolbased survey in China. Eur J Public Health 24, 880-887.

10. Qian L, Zhang F, Newman IM et al. (2017) Effects of selected socio-demographic characteristics on nutrition knowledge and eating behavior of elementary students in two provinces in China. BMC Public Health 18, 21.

11. Slusser W, Prelip M, Kinsler J et al. (2011) Challenges to parent nutrition education: a qualitative study of parents of urban children attending low-income schools. Public Health Nutr 14, 1833-1841.

12. Cockerham WC (2005) Health lifestyle theory and the convergence of agency and structure. J Health Soc Behav $\mathbf{4 6}$, 51-67.

13. Anderson J (2014) The impact of family structure on the health of children: effects of divorce. Linacre $Q \mathbf{8 1}, 378-387$.

14. Fagan PF \& Churchill A (2012) The effects of divorce on children. Marri Research. https://www.frc.org/EF/EF12A22.pdf (accessed March 2020).

15. Shibeshi A (2015) Causes of Divorce and Its Effects on Children's Wellbeing in Yeka Sub-city. PhD Thesis. Ethiopia, Addis Ababa: Addis Ababa University.

16. Bernardi F \& Boertien D (2017) Non-intact families and diverging educational destinies: a decomposition analysis for Germany, Italy, the United Kingdom and the United States. Soc Sci Res 63, 181-191.

17. Kleinsorge C \& Covitz LM (2012) Impact of divorce on children: developmental considerations. Pediatr Rev 33, 147-154, quiz 154-155.

18. Edwards AN (2014) Dynamics of Economic Well-being: Poverty, 2009-2011. Current Population Reports, P70-137, U.S. Census Bureau, Washington, DC. http://www.census. gov/prod/2014pubs/p70-137.pdf (accessed March 2020).

19. Formisano A, Hunsberger M, Bammann K et al. (2013) Family structure and childhood obesity: results of the IDEFICS Project. Public Health Nutr 17, 2307-2315.

20. Mauskopf SS, O'Leary AK, Banihashemi A et al. (2015) Divorce and eating behaviors: a 5-day within-subject study of preadolescent obesity risk. Child Obes 11, 122-129.

21. Health Behaviour in School-aged Children: World Health Organization Collaborative Cross-National Survey (2020). http://www.hbsc.org (accessed March 2020).

22. Health Behaviour in School-aged Children Study: A World Health Organization Collaborative Cross-National Study (2013). Internal Protocol 2013/2014. Scotland: University of St. Andrews. https://drive.google.com/file/d/0BxYI8UzU8n1 faEZ4WUhmSOUxcVU/view (accessed March 2020).

23. Currie C, Molcho M, Boyce W et al. (2008) Researching health inequalities in adolescents: the development of the Health Behaviour in School-Aged Children (HBSC) family affluence scale. Soc Sci Med 66, 1429-1436.

24. Torsheim T, Cavallo F, Levin KA et al. (2016) Psychometric validation of the revised Family Affluence Scale: a latent variable approach. Child Indic Res 9, 771-784.

25. Kunst AE \& Mackenbach JP (1995) Measuring Socioeconomic Inequalities in Health. Copenhagen: World Health Organisation, Regional Office for Europe.

26. Zaborskis A \& Grincaite M (2018) Gender and age differences in social inequality on adolescent life satisfaction: a comparative analysis of health behaviour data from 41 countries. Int J Environ Res Public Health 15, 1297. 
27. Zaborskis A, Grincaite M, Lenzi M et al. (2019) Social inequality in adolescent life satisfaction: comparison of measure approaches and correlation with macrolevel indices in 41 countries. Soc Indic Res 141, 1055-1079.

28. Čekanavičius V \& Murauskas G (2009). Statistika ir jos taikymai (Statistics and Its Applications), 3 knyga. Vilnius: REV UAB BI (In Lithuanian).

29. Arbuckle JL (2012) IBM SPSS AMOS 21 User's Guide. New York: Amos Development Corporation.

30. Hooper D, Caughlan J \& Mullen MR (2008) Structural equation modeling: guidelines for determining model fit. Electr $J$ Bus Res Methods 6, 53-60.

31. Costa BVL, Menezes MC, Oliveira CDL et al. (2019) Does access to healthy food vary according to socioeconomic status and to food store type? An ecologic study. BMC Public Health 19, 775 .

32. Pearson N, Biddle SJ \& Gorely T (2009) Family correlates of fruit and vegetable consumption in children and adolescents: a systematic review. Public Health Nutr 12, 267-283.

33. Hilsen M, van Stralen MM, Klepp K-I et al. (2011) Changes in 10-12-year old's fruit and vegetable intake in Norway from 2001 to 2008 in relation to gender and socioeconomic status - a comparison of two cross-sectional groups. Int J Behav Nutr Phys Act 8, 108.

34. Rasmussen M, Krølner R, Klepp KI et al. (2006) Determinants of fruit and vegetable consumption among children and adolescents: a review of the literature. Part I: quantitative studies. Int J Behav Nutr Phys Act 3, 22.

35. Rasmussen M, Pedersen TP, Johnsen NF et al. (2018) Persistent social inequality in low intake of vegetables among adolescents, 2002-2014. Public Health Nutr 21, 1649-1653.

36. Pearson N, Atkin AJ, Biddle SJ et al. (2010) Parenting styles, family structure and adolescent dietary behaviour. Public Health Nutr 13, 1245-1253.

37. Cullen KW, Baranowski T, Rittenberry L et al. (2000) Socioenvironmental influences on children's fruit, juice and vegetable consumption as reported by parents: reliability and validity of measures. Public Health Nutr 3, 345-356.

38. Kremer-Sadlik T, Morgenstern A, Peters C et al. (2015) Eating fruits and vegetables. An ethnographic study of American and French family dinners. Appetite 89, 84-92.

39. Gaspar de Matos M, Palmeira AL, Gaspar T et al. (2016) Social support influences on eating awareness in children and adolescents: the mediating effect of self-regulatory strategies. Glob Public Health 11, 437-448.

40. Lazzeri G, Ahluwalia N, Niclasen B et al. (2016) Trends from 2002 to 2010 in daily breakfast consumption and its sociodemographic correlates in adolescents across 31 countries participating in the HBSC study. PLOS One 11, 0151052.

41. Currie C, Nic Gabhainn S, Godeau E et al. (editors) (2008) Inequalities in Young People's Health: HBSC International Report from the 2005/2006 Survey. Copenhagen: World Health Organization Regional Office for Europe.

42. Mitchell AJ (editor) (1990) Formulation and Production of Carbonated Soft Drinks. London: Blackie and Son Ltd.

43. Myszkowska-Ryciak J, Harton A, Lange E et al. (2019) Nutritional behaviors of polish adolescents: results of the wise nutrition-healthy generation project. Nutrients 11, 1592.

44. Lipsky LM, Nansel TR, Haynie DL et al. (2017) Diet quality of US adolescents during the transition to adulthood: changes and predictors. Am J Clin Nutr 105, 1424-1432.

45. Pettigrew S, Jongenelis M, Miller C et al. (2017) A path analysis model of factors influencing children's requests for unhealthy foods. Eating Behav 24, 95-101.

46. Adamo KB, Papadakis S, Dojeiji L et al. (2010) Using path analysis to understand parents' perceptions of their children's weight, physical activity and eating habits in the Champlain region of Ontario. Paediatr Child Health 15, e33-e41.

47. Moore GF, Tapper K, Moore L et al. (2008) Cognitive, behavioral, and social factors are associated with bias in dietary questionnaire self-reports by schoolchildren aged 9 to 11 years. J Am Diet Assoc 108, 1865-1873. 